\title{
The Sunspots
}

\author{
Angel Fierros Palacios \\ Instituto de Investigaciones Eléctricas, División de Energías Alternas, Mexico City, México \\ Email: afierros@iie.org.mx
}

Received 4 June 2015; accepted 16 October 2015; published 19 October 2015

Copyright (C) 2015 by author and Scientific Research Publishing Inc.

This work is licensed under the Creative Commons Attribution International License (CC BY).

http://creativecommons.org/licenses/by/4.0/

(c) (i) Open Access

\begin{abstract}
In the present paper, the theoretical frame work of magneto hydrodynamics (MHD) is used to give a solution of the problem about the origin, persistence and disappearance of the Sunspots; as well as their tendency to appear as bipolar magnetic couples. According to the results obtained, a possible explanation about the change of polarity in both solar hemispheres is given. Heuristic but logical arguments about the periodicity of the phenomenon of the observed magnetic polarity and the tendency of couples of Sunspots to appear solely in certain latitudes that can be called tropical regions of the Sun are presented. Finally, an indirect experimental test is proposed to show the possible process that produces the polarity of the Sunspots in a given cycle, as well as the invertion of that polarity in the next solar cycle.
\end{abstract}

\section{Keywords}

Magnetic Field of the Spots, Sunspots' Theory, Magnetic Regulation of Thermal Convection, Polarity of the Sunspots Couples, Solar Cycle, Structure and Stability of the Spots, Life Time of the Spots

\section{Introduction}

The sun is a huge concentration of fluid at very high temperature, whose parts are kept together and in dynamic equilibrium with fluid's gas pressure force by gravitational attraction. They are also under the influence of a self-generated magnetic field. Careful observation of the solar surface shows a granular texture given by shortlived objects known as granules, somewhat brighter than their neighbors. Also easily observed are the so called Sunspots which are colder and darker regions than the solar photosphere; discovered first by Chinese astronomers, then by Galileo and observed by himself and his contemporaries. These objects have a somewhat nebulous origin along with very peculiar properties and general behaviour, which leads to assume that under the surface of sun's photosphere, certain local processes take place destabilizing it and creating the phenomenon.

The present work focuses on the study of the thermal energy conditions which create a thermal instability in 
some regions of the photosphere, and their relationship with the appearance, properties and life time of the Sunspots. A large amount of observational data has been accumulated of the subject [1]; in particular, it is well known that the presence of Sunspots on the solar disk is related to a substantial increase in sun's activity. The most important expression of that activity is the emission of large quantities of charged particles which conforms the so called solar wind. The strong interaction of the solar wind with earth's magnetic field and the ionosphere produces on one side the gigantic and most beautiful Aurora Borialis; and on the other side, serious perturbations of the telecommunications systems.

The vast majority of the Sunspots are observed in certain regions of the sun. They appear predominantly in two stripes of equal latitude to the North and South of the Solar Equator in which can be called the tropical regions. They have the tendency to appear in big groups or in couples; in each of which its two members always have opposite magnetic polarity.

A typical Sunspot has the following observed structure and dimensions. It possesses a dark nucleus called Umbra of about 18,000 km in diameter, and a somewhat lighter halo called Penumbra, 20,000 km wide. The lower luminosity of the spot as compared to the photosphere is due to a decrease in its temperature.

A remarkable fact that should be noted is that the polarity of the Sunspot couples in the northern hemisphere is always opposite to the polarity of the Sunspot couples in the southern hemisphere. This disparity changes periodically with a period of about 11 years.

This problem is similar to that of the mechanical instability which occurs in some regions of the terrestrial atmosphere [2] [3]. It is known that the mechanical equilibrium is broken in this case when the following conditions are fulfilled in the air, considered as a viscous and compressible fluid which moves in a gravitational field. The temperature distribution is only a function of the height above ground level and the temperature gradient in the vertical direction is directed downward and its magnitude is greater than a certain value [2].

The non existence of mechanical equilibrium leads to the appearance a certain movement in the fluid; internal currents appear mixing the fluid until its temperature is uniform and reach a constant value in all its volume. This type of movement of a fluid in a gravitational field receives the name of Free or Thermal Convection [3]. It is known that in the case of the Sunspots, the fluid is under the influence of an intense magnetic field [4]. This fact makes difficult the mathematical treatment of the problem because it deals with a complicated interaction between the flow of compressible and conducting viscous fluid, and the electromagnetic phenomena deriving from the influence of the external magnetic field. It is well known, that this interaction should be treated with the help of the balance equations of fluid dynamics and Maxwell equations of classical electrodynamics [5] [6]; that is, with the assistance of the magnetohydrodynamic equations [5] [7].

A thermal instability which triggers convection currents can be produced in any real fluid with the above mentioned characteristics, as long as the fluid is exposed to non uniform heating from below [4]. Nevertheless, this tendency to instability can be substantially depressed when dealing with a real conducting fluid under the influence of an intense magnetic field, which gives the fluid some kinds of magnetic viscosity and a certain degree of stiffness [4]. This is an important fact because if the magnetic forces generated in a sunspot can substantially reduce convective movements, such reduction must take place at the expense of the thermal energy in those regions, resulting in a cold and dark spot due to the energy decrease [4]. In any case, the persistence of the magnetic field in the Sunspots proves the existence there of a magnetic mechanism for the regulation of thermal convection; this process has the mission to delay as much as possible the onset of the starting mechanism for convective movements [2] [3].

\section{Dynamic Equilibrium between Regulatory and Startup Mechanisms of Convection}

For magnetomechanical equilibrium to be reached in any region of the solar photosphere, it is necessary that (see Appendix)

$$
\operatorname{grad}\left(p-\frac{H^{2}}{8 \pi}\right)=\rho \boldsymbol{g}
$$

where $p(\rho, T, \boldsymbol{H})$ is the hydrostatic pressure and $H^{2} / 8 \pi$ the hydrostatic magnetic pressure [5] [7]; $\boldsymbol{H}(\boldsymbol{x}, t)$ represents the magnetic field in that region, $\rho(x, t)$ the mass density of the solar fluid and $\boldsymbol{g}$ the acceleration 
of gravity at the surface of the sun.

If the Z-axis of the reference inertial frame used points vertically upwards, Equation (1) can be written as

$$
\frac{\mathrm{d} p}{\mathrm{~d} z}=-\frac{g}{V}+\operatorname{grad}_{z}\left(\frac{H^{2}}{8 \pi}\right)
$$

where $V=1 / \rho$ is the specific volume.

It is known that if temperature is not a constant throughout a fluid, the resulting mechanical equilibrium could be stable or unstable depending of certain conditions [2]. In the case of the Sunspots, it is necessary to determine the conditions under which the instability could exist in those regions and to find out its consequences.

The theoretical treatment for the adiabatic displacement of a mass of solar fluid in the photosphere is identical to the one given for an air mass adiabatically displaced in the terrestrial atmosphere [2] [3]. The general condition of stability which is obtained in both cases is the same; that is, the specific entropy of the system increases with altitude, thus [2] [3]

$$
\frac{\mathrm{d} s}{\mathrm{~d} z}>0 .
$$

It can be shown that for both situations, the previous conditions can be written as follows [2] [3]

$$
\frac{\mathrm{d} s}{\mathrm{~d} z}=\frac{C_{P}}{T} \frac{\mathrm{d} T}{\mathrm{~d} z}-\left(\frac{\partial V}{\partial T}\right)_{p} \frac{\mathrm{d} p}{\mathrm{~d} z}>0,
$$

where $c_{P}$ is the specific heat of the fluid at a constant pressure and $T$ its temperature. Then, according to Equation (2), from (4) we have that

$$
\operatorname{grad}_{z} H^{2}>\frac{8 \pi c_{P} \operatorname{grad}_{z} T}{T\left(\frac{\partial V}{\partial T}\right)_{p}}+\frac{8 \pi g}{V}
$$

In this case, the condition to be satisfied so that a thermal instability can be produced in some regions of the solar photosphere and strongly regulated by the magnetic field that generates the Sunspots can be given as follows:

$$
\left|\operatorname{grad}_{z} H^{2}\right|>\frac{8 \pi c_{P}\left|\operatorname{grad}_{z} T\right|}{T\left(\frac{\partial V}{\partial T}\right)_{p}}+\frac{8 \pi g}{V} .
$$

In other words, the onset of convective movements in those regions can be substantially delayed if the magnitude of the Z-component of the gradient of the square of the existing magnetic field is larger than a certain value. The above condition must be fulfilled so that the thermal convection in the spots is magnetically regulated. It simply means that the persistence of the magnetic field in those regions, assures the permanency in time of the Sunspots in the solar disk. In fact as the magnetic field fades away, the mechanism of magnetic regulation weakens, allowing the thermal processes to take over the situation; as a result, the mechanism for the onset of convection begins generating and becomes more dominant so that, when $\boldsymbol{H}=0$, convective movements are produced which mix the fluid of the spots with the surrounding fluid until the temperature becomes uniform. At that point the sunspots disappear and the solar activity comes to an end in those regions. Therefore, free convection is produced when the magnitude of the temperature gradient in the Z-direction is greater than a certain value [2] [3]; this is, when

$$
\left(\frac { \mathrm { d } T } { \mathrm { d } z } \left(>\frac{g T}{V c_{P}}\left(\frac{\partial V}{\partial T}\right)_{p}\right.\right.
$$

as is easily seen from (6) when $\boldsymbol{H}=0$.

From Thermodynamics we have that [2] [3]

$$
c_{p}\left(\frac{\partial V}{\partial s}\right)_{p}=T\left(\frac{\partial V}{\partial T}\right)_{p}
$$


Substituting this relation in (5), thereafter integrating the resultant expression and in the result obtained Equation (8) is used again, it is easy to see that

$$
H^{2}>\frac{8 \pi c_{P}}{\left(\frac{\partial V}{\partial T}\right)_{p}}+\frac{8 \pi g z}{V}
$$

where $z$ is some characteristic height in the photosphere where the Sunspots are produced. Now, consider that

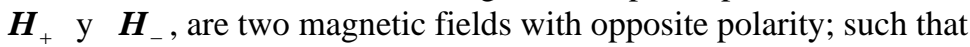

$$
\boldsymbol{H}_{+} \cdot \boldsymbol{H}_{-}=H^{2} .
$$

Furthermore,

$$
Q_{+}=\left[\frac{8 \pi c_{P}}{\left(\frac{\partial V}{\partial T}\right)_{p}}+\frac{8 \pi g z}{V}\right]^{1 / 2}
$$

and

$$
Q_{-}=-\left[\frac{8 \pi c_{p}}{\left(\frac{\partial V}{\partial T}\right)_{p}}+\frac{8 \pi g z}{V}\right]^{1 / 2}
$$

so that with (10) and the product of these two numbers the relation (9) is recovered. Thus, the persistence of each couple of sunspots on Sun's surface as assured when the magnitude of the magnetic field which generates and maintains them is greater than a certain amount, that is

$$
\left|\boldsymbol{H}_{+}\right|>2\left[\frac{2 \pi c_{P}}{\left(\frac{\partial V}{\partial T}\right)_{p}}+\frac{2 \pi g z}{V}\right]^{1 / 2}
$$

and

$$
\left|\boldsymbol{H}_{-}\right|>2\left[\frac{2 \pi c_{P}}{\left(\frac{\partial V}{\partial T}\right)_{p}}+\frac{2 \pi g z}{V}\right]^{1 / 2}
$$

Clearly, both magnetic fields have the same magnitude. It can further be reasonably assumed that beyond sharing the previous quality, they also have the same direction. Thus, if for example $\boldsymbol{H}_{+}$is a magnetic field with the (N-S) polarity; $\boldsymbol{H}_{-}$will be a magnetic field with opposite polarity; that is (S-N). Then, both magnetic fields have the same magnitude and direction but opposite polarities. This means that, couples of regions darker and colder rather than their surroundings, with opposite magnetic polarities and closely related between themselves must appear in the solar photosphere; one, where the lines of force of the magnetic field points outwards from Sun's surface; and another, where they point inwards. In other words, the Sunspots must appear at the same heliographic latitude and in the form of magnetic bipolar couples; in full agreement with the results of astronomical observations [4].

\section{The Velocity of the Fluid in the Sunspots}

Consider a huge mass of viscous compressible and conducting fluid, which moves in the presence of a magnetic field and is under the influence of a gravitational field. Let $T(x, t)=T_{o}+T^{\prime}$ be the corresponding field of 
temperatures in the photosphere, $T_{o}$ the temperature of the Sunspots and $T^{\prime}$ the temperature at which the dynamic equilibrium between the mechanisms of magnetic regulation and the onset of thermal convection is broken, so that $T^{\prime} \ll T_{o}$. Let $\rho(x, t)=\rho_{o}+\rho^{\prime}$ be the mass density of the solar fluid in the photosphere, with $\rho_{o} \gg \rho^{\prime}$ the mass density of the fluid in the Sunspots and $\rho^{\prime}$ the change in density while the mechanism for the onset of convection develops. Since both $T^{\prime}$ and $\rho^{\prime}$ are small quantities, speaking in relative terms, it can be said that [2] [3]

$$
\rho^{\prime}=\left(\frac{\partial \rho}{\partial T}\right)_{p} T^{\prime}=-\rho_{o} \alpha T^{\prime}
$$

where the proposed decomposition for density was used. Furthermore, by definition,

$$
\alpha \equiv-\frac{1}{\rho}\left(\frac{\partial \rho}{\partial T}\right)_{p}
$$

is the thermal expansion coefficient of the conductor fluid. With respect to hydrostatic pressure we have that $p(x, t)=p_{o}+p^{\prime}$, with $p_{o} \gg p^{\prime}$. However, $p_{o}$ is not a constant, since it represents the pressure corresponding to the dynamic equilibrium in the inner regions of the Sunspots whose temperatures and densities are constant and equal to $T_{o}$ and $\rho_{o}$, respectively. Since the fluid is under to the gravitational attraction of the Sun, it will be assumed that $p_{o}$ varies with height according to the hydrostatic equation [2] [3]

$$
p_{o}=\rho_{o} \boldsymbol{g} \cdot \boldsymbol{x}+\text { constant }
$$

Consider now the equation of movement of MHD, or generalized Cauchy equation [5] [7]

$$
\rho \frac{\mathrm{d} \boldsymbol{v}}{\mathrm{d} t}-\operatorname{div} \tilde{\sigma}^{o}-\rho \boldsymbol{f}=0
$$

where $\boldsymbol{f}$ is the body force per unit mass, which in this case is equal to $\boldsymbol{g}$, the acceleration of gravity at the surface of the sun [2]. Besides,

$$
\sigma_{i j}^{o}=-p \delta_{i j}+\sigma_{i j}^{\prime}+\frac{1}{4 \pi}\left[H_{i} H_{j}-\frac{1}{2} H^{2} \delta_{i j}\right]
$$

Are the components of the generalized stress tensor [7], $\delta_{i j}$ the components of Kronecker's delta and the third term of the right hand side of Equation (19) one has the components of Maxwell's magnetic stress tensor [5] [7]. On the other hand,

$$
\sigma_{i j}^{\prime}=\eta\left[\frac{\partial v^{i}}{\partial x^{j}}+\frac{\partial v^{j}}{\partial x^{i}}-\frac{2}{3} \delta_{i j} \frac{\partial v^{\ell}}{\partial x^{\ell}}\right]+\zeta \delta_{i j} \frac{\partial v^{\ell}}{\partial x^{\ell}}
$$

are the components of the viscosity stress tensor [6]. In this expression $\eta$ and $\zeta$ are the coefficients of viscosity which in general are functions of pressure and temperature, but will be considered constants for the mechanisms of magnetic regulation and the onset of thermal convection. Now, substituting (19) into (18) one gets

$$
\frac{\mathrm{d} \boldsymbol{v}}{\mathrm{d} t}=-\frac{1}{\rho_{o}} \boldsymbol{g r a d} p-\frac{1}{4 \pi \rho_{o}} \boldsymbol{H} \times \boldsymbol{r o t} \boldsymbol{H}+\boldsymbol{g}+\boldsymbol{f}_{v}
$$

where

$$
\boldsymbol{f}_{v}=\frac{1}{\rho_{o}} \operatorname{div} \tilde{\sigma}^{\prime}
$$

is the specific viscous force and the following vectorial identity has been used

$$
(\boldsymbol{H} \cdot \operatorname{grad}) \boldsymbol{H}-\frac{1}{2} \operatorname{grad} H^{2}=-\boldsymbol{H} \times \boldsymbol{r o t} \boldsymbol{H}
$$

On the other hand,

$$
\frac{1}{\rho} \operatorname{grad} p=\frac{\operatorname{grad} p_{o}}{\rho_{o}}+\frac{\boldsymbol{g r a d} p^{\prime}}{\rho_{o}}-\frac{\operatorname{grad} p_{o}}{\rho_{o}^{2}} \rho^{\prime}
$$


where calculation up to the first order term for the small quantities was performed. Now, from (17) is clear that

$$
\frac{1}{\rho_{o}} \operatorname{grad} p_{o}=\boldsymbol{g}
$$

and from (15)

$$
\frac{\rho^{\prime}}{\rho_{o}^{2}} \operatorname{grad} p_{o}=-\alpha \boldsymbol{g} T^{\prime}
$$

On the other hand, consider that $\boldsymbol{H}(\boldsymbol{x}, t)=\boldsymbol{H}_{o}+\boldsymbol{h}, \boldsymbol{H}_{o} \gg \boldsymbol{h}$ being a uniform magnetic field, $\boldsymbol{H}(\boldsymbol{x}, t)$ the external magnetic field and $\boldsymbol{h}$ an small perturbation [4]. Then, from (21) the following is obtained

$$
\frac{\mathrm{d} \boldsymbol{v}}{\mathrm{d} t}=-\frac{1}{\rho_{o}} \boldsymbol{g r a d} p^{\prime}-\alpha \boldsymbol{g} T^{\prime}-\frac{1}{4 \pi \rho_{o}} \boldsymbol{H}_{o} \times \boldsymbol{r o t h}+\boldsymbol{f}_{v}
$$

Because is clear from the previous paragraph that $\boldsymbol{\operatorname { r o t }} \boldsymbol{H}_{o}=0$. Now, an equation is required which relates pressure with density, temperature and magnetic field. Such a relation is the MHD thermal equation of state, which in its most general form is given by the following expression [7]

$$
p=c_{o}^{2} \rho+\frac{\beta}{k} T+\frac{H^{2}}{8 \pi}
$$

where, $c_{o}^{2}$ is the square of the velocity of sound in the medium, $\beta$ is the volumetric expansion coefficient and $k$ the isometric compressibility of the fluid under consideration [7]. On the other hand, from the scalar equation for mass density [8]

$$
\rho(\boldsymbol{x}, t)=\frac{\rho_{o}}{J} \boldsymbol{u} \cdot \operatorname{grad} J
$$

where $\boldsymbol{u}(\boldsymbol{x})$ is the displacement vector and $J$ the Jacobian of the transformation [8] [9], the following is obtained

$$
\rho^{\prime}=\rho_{o}\left[\frac{\mathbf{u}}{J} \cdot \operatorname{grad} J-1\right]
$$

so that, according to (15)

$$
\alpha=\frac{1}{T^{\prime}}\left[1-\frac{\boldsymbol{u}}{J} \cdot \operatorname{grad} J\right]
$$

With (15) into (28) and with the approximations already made, it is easy to see that

$$
p^{\prime}=-\rho_{o} \gamma T^{\prime}+\frac{h^{2}}{8 \pi}-p_{o}+c_{o}^{2} \rho_{o}+\frac{\beta}{k} T_{o}+\frac{H_{o}^{2}}{8 \pi}+\frac{\boldsymbol{H}_{o} \cdot \boldsymbol{h}}{4 \pi}
$$

where

$$
\gamma \equiv \alpha c_{o}^{2}-\frac{\beta}{k \rho_{o}}
$$

is a constant. On the other hand, for the equilibrium situation between the regulatory and startup mechanisms we have that

$$
p_{o}=c_{o}^{2} \rho_{o}+\frac{\beta}{k} T_{o}+\frac{H_{o}^{2}}{8 \pi}
$$

only, because according the approximations already made, $H^{2} \gg \boldsymbol{H}_{o} \cdot \boldsymbol{h}$. Furthermore,

$$
p^{\prime}=-\rho_{o} \gamma T^{\prime}+\frac{h^{2}}{8 \pi}
$$

is the explicit form of the required thermal equation of state. From this result, the following expression can be 
obtained

$$
-\frac{1}{\rho_{o}} \operatorname{grad} p^{\prime}=\gamma \operatorname{grad} T^{\prime}-\operatorname{grad} p_{M}^{o}
$$

Then, from relation (26) the following result is obtained

$$
\frac{\mathrm{d} \boldsymbol{v}}{\mathrm{d} t}=\gamma \boldsymbol{g r a d} T^{\prime}-\alpha \boldsymbol{g} T^{\prime}-\boldsymbol{g r a d} p_{M}^{o}-\frac{\boldsymbol{H}_{o}}{4 \pi \rho_{o}} \times \boldsymbol{r o t h}+\boldsymbol{f}_{v}
$$

where

$$
p_{M}^{o} \equiv \frac{h^{2}}{8 \pi \rho_{o}} .
$$

On the other hand, the magnetic field of the Sunspots should satisfy the following field equation [5]

$$
\operatorname{roth}=\frac{4 \pi}{c} \boldsymbol{j}
$$

where $\boldsymbol{j}(\boldsymbol{x}, t)$ is the conduction current density and $c$ the velocity of light in the empty space. Nevertheless, to study the flow of a real conducting fluid, which moves in the presence of an external magnetic field, one must recognize that it does not conduct any current. In this case, the previous relationship is reduced to roth $=0$, since $\boldsymbol{j}=0$. Then, in (36) we get

$$
\frac{\mathrm{d} \boldsymbol{v}}{\mathrm{d} t}=\gamma \operatorname{grad} T^{\prime}-\alpha \boldsymbol{g} T^{\prime}-\boldsymbol{g r a d} p_{M}^{o}+\boldsymbol{f}_{v}
$$

From the previous expression it is possible to determine the magnetohydrodynamic force which is responsible for the dynamic equilibrium between the onset of thermal convection and the mechanism of magnetic regulation, that is

$$
\boldsymbol{F}_{M H D}=\gamma \operatorname{grad} T^{\prime}-\operatorname{grad} p_{M}^{o}
$$

Consider now that for the condition of dynamic equilibrium, the viscous force is not relevant so that in (39) the term $\boldsymbol{f}_{v}$ can be ignored. This approximation is possible due to the following. While conditions (13) and (14) are fulfilled, the dominant mechanism is the magnetic regulation of thermal convection and the convective movements are controlled, and clearly, sufficiently diminished; so that it is reasonable to assume that the viscous force can be ignored in comparison with the MHD force (40) and with the term which contains sun's acceleration of gravity. As mentioned in the introductory section, the presence of an intense magnetic field in the Sunspots confers the fluid a certain rigidity which makes the movement difficult in those regions; so that it is possible to assume that the velocity of the fluid in the Sunspots is small compared with the velocity of the warmer fluid found in their surroundings. In consequence, if the velocity field inside the Spots is small, relatively speaking, and the distances dealt with are very large, the velocity gradients should be even smaller. Since $\boldsymbol{f}_{v}$ is dependent on the gradients of the velocity field, then it is expected to be a small quantity in comparison with the other terms. When the magnetic field starts to decay and weakens, the starter mechanism of thermal convection is initiated. Nevertheless, it can be said that while the dynamic equilibrium between both mechanisms exists, the conditions to produce convective movements have been arising very slowly; so that it is possible to assert that the change in the temperature of the Sunspots from $T_{o}$ to $T$, occur in the steady state. Then the temperature $T^{\prime}$ at which the dynamic equilibrium is broken and convection starts, depends only on the coordinates and not on the time. In consequence, throughout the whole life of the Sunspots the viscous force is not important and it can be ignored for the whole analysis of the problem.

Once the equilibrium is broken, convective movements are rapidly established mixing the fluid of the Sunspots with that of its surroundings until the average temperature $T$ of the photosphere is reached. It is possible that during this process, the viscosity of the medium, and in consequence of the viscous force, becomes very important; but at this time, the Sunspots have disappeared because the magnetic field which generates them is zero and, clearly, the thermal gradient becomes null and, hence, the solar activity in those regions comes to an end. Since the remaining terms of the relation (39) are not explicitly depending on time, that equation can be integrated to obtain 


$$
\boldsymbol{v}^{\prime}=\boldsymbol{v}_{o}+\left[\gamma \operatorname{grad} T^{\prime}-\alpha \boldsymbol{g} T^{\prime}-\operatorname{grad} p_{M}^{o}\right]\left(t^{\prime}-t_{o}\right)
$$

However, $\boldsymbol{v}_{o}=0$ because it is the average fluid velocity inside the spots corresponding to the dynamic equilibrium, when the magnetic field that generates them reaches its maximum value and the convective movements are magnetically regulated and very depressed; that is, in the mature stage of the spots. Under this situation, it can be said that $\boldsymbol{v}_{o}$ is the vector addition of the possible fluid movements inside those regions and that such vector sum is zero. On the other hand, even when the dynamic equilibrium between the mentioned mechanisms may exist and the movement of the fluid in the central regions of the spots may be very depressed; there will be great activity at its boundaries. Actually the fluid there overflows diverging from the center to the edge with a movement which is practically parallel to the surface of the Sun, due to the gravity pull. This is due to the combination of two effects; one, the dominant effect of the solar gravity, and the other, the fact that at the edges of the spots the control of thermal convection by the mechanism of magnetic regulation is not complete enough; so that there exists a residual influence of the thermal gradient over the gradient of the magnetic hydrostatic pressure. Thus, and due to the effect of thermal stirring, the fluid flows through the edges of the Spot at a certain velocity $\boldsymbol{v}^{\prime}$, but it almost immediately collapses towards the surface of the sun and under the pull of its gravity, giving the spots their characteristic appearance [10]. On the other hand, the thermal fluctuations which are likely to occur at the edges, should give origin to large splashing of fluid which raise to a great altitude over the photosphere, probably constituting the so called Faculae which are the usual companions of the spots. The overflow of the fluid in those regions is very similar to what occurs when a viscous liquid boils and overflows over the edges of its container. In such process plashing is also very common. In any case when $\boldsymbol{v}_{o}=0$ and the scale of time is chosen so that $t_{o}=0$, one has that previous expression

$$
\boldsymbol{v}^{\prime}=\left[\gamma \boldsymbol{g r a d} T^{\prime}-\alpha \boldsymbol{g} T^{\prime}-\boldsymbol{g r a d} p_{M}^{o}\right] t^{\prime}
$$

If only the $Z$ direction is considered, the following result is obtained

$$
v_{z}^{\prime}=\left[\gamma \frac{\mathrm{d} T^{\prime}}{\mathrm{d} z}+\alpha g T^{\prime}-\frac{\mathrm{d} p_{M}^{o}}{\mathrm{~d} z}\right] t^{\prime}
$$

When the onset mechanism of the thermal convection begins to dominate the phenomenon, the magnetic part begins to decrease so that the term $\gamma \boldsymbol{g r a d} T^{\prime}$ takes over of the situation. In the limit when $\boldsymbol{h}=0$ the vectorial velocity at which the convective movements begins is obtained in (42); this is

$$
\boldsymbol{v}_{c}^{\prime}=\left(\gamma \operatorname{grad} T^{\prime}-\alpha \boldsymbol{g} T^{\prime}\right) t^{\prime}
$$

and, its vertical component clearly is

$$
v_{z c}^{\prime}=\left(\gamma \frac{\mathrm{d} T^{\prime}}{\mathrm{d} z}+\alpha \boldsymbol{g} T^{\prime}\right) t^{\prime}
$$

where the subscript $c$ indicates that the process of thermal convection is being considered.

\section{The General Equation for Heat Transfer}

So far the velocity field and the MHD force responsible for the dynamic equilibrium have been calculated. Also available is the scalar equation for the mass density, which is valid for any fluid, and of course, the thermal equation of state for the phenomenon of the solar spots. The general equation of heat transfer remains missing in order to have an analytical solution to the problem of origin, duration and disappearance of the spots. This last equation can be obtained from the law of energy conservation for MHD [7]; which for any viscous conducting and compressible fluid, that moves in the presence of a magnetic field is given by

$$
\begin{aligned}
& \frac{\partial}{\partial t}\left(\frac{1}{2} \rho v^{2}+\rho \varepsilon+\frac{H^{2}}{8 \pi}\right) \\
& =-\operatorname{div}\left[\rho \boldsymbol{v}\left(\frac{1}{2} v^{2}+\omega^{\prime}\right)+\frac{\boldsymbol{H}}{4 \pi} \times(\boldsymbol{v} \times \boldsymbol{H})-\frac{c^{2}}{16 \pi^{2} \sigma} \boldsymbol{H} \times \boldsymbol{r o t} \boldsymbol{H}-\boldsymbol{v} \cdot \tilde{\sigma}^{\prime}-\operatorname{\kappa grad} T\right]
\end{aligned}
$$

where $\omega^{\prime}=\varepsilon+p / \rho+H^{2} / 8 \pi \rho$ is the generalized specific enthalpy, $\kappa$ the coefficient of thermal conductivity 
and $\sigma$ the electrical conductivity of the continuous medium [5] [7]. Besides, $\varepsilon$ is the specific internal energy of the solar fluid. If the left member of (46) is developed, it can be shown that [2]

$$
\begin{aligned}
\frac{\partial}{\partial t}\left(\frac{1}{2} \rho v^{2}+\rho \varepsilon+\frac{H^{2}}{8 \pi}\right)= & -\operatorname{div}\left[\rho \boldsymbol{v}\left(\frac{1}{2} v^{2}+\omega\right)\right]+\rho \boldsymbol{v} \cdot \frac{\partial \boldsymbol{v}}{\partial t}+\rho T \frac{\mathrm{d} s}{\mathrm{~d} t}+\boldsymbol{v} \cdot \operatorname{grad} p \\
& +\rho \boldsymbol{v} \cdot \operatorname{grad}\left(\frac{1}{2} v^{2}\right)+\frac{1}{4 \pi} \boldsymbol{H} \cdot \frac{\partial \boldsymbol{H}}{\partial t}
\end{aligned}
$$

where $\omega=\varepsilon+p / \rho$ is the usual specific enthalpy. On the other hand [7],

$$
\frac{1}{4 \pi} \boldsymbol{H} \cdot \frac{\partial \boldsymbol{H}}{\partial t}=\frac{1}{4 \pi} \boldsymbol{H} \cdot[\nabla \times(\boldsymbol{v} \times \boldsymbol{H})]+\frac{c^{2}}{16 \pi^{2} \sigma} \boldsymbol{H} \cdot\left(\nabla^{2} \boldsymbol{H}\right)
$$

Then from Navier-Stokes equations for MHD in the absence of body forces

$$
\rho \frac{\partial \boldsymbol{v}}{\partial t}=-\rho(\boldsymbol{v} \cdot \operatorname{grad}) \boldsymbol{v}-\boldsymbol{g r a d}\left(p+\frac{H^{2}}{8 \pi}\right)+\frac{1}{4 \pi}(\boldsymbol{H} \cdot \operatorname{grad}) \boldsymbol{H}+\boldsymbol{F}_{v}
$$

where

$$
\boldsymbol{F}_{v}=\operatorname{div} \tilde{\sigma}^{\prime}
$$

is the viscous force, with $\tilde{\sigma}^{\prime}$ the viscosity stress tensor of Navier-Stokes [3]; the following is obtained

$$
\rho \boldsymbol{v} \cdot \frac{\partial \boldsymbol{v}}{\partial t}=-\rho \boldsymbol{v} \cdot \boldsymbol{g r a d}\left(\frac{1}{2} v^{2}\right)-\boldsymbol{v} \cdot \boldsymbol{g r a d} p-\operatorname{div}\left(\frac{H^{2}}{8 \pi} \boldsymbol{v}\right)+\boldsymbol{v} \cdot \boldsymbol{F}_{v}
$$

because

$$
\boldsymbol{v} \cdot \operatorname{grad}\left(\frac{H^{2}}{8 \pi}\right)+\frac{\boldsymbol{v}}{4 \pi} \cdot[(\boldsymbol{H} \cdot \operatorname{grad}) \boldsymbol{H}]=\operatorname{div}\left(\frac{H^{2}}{8 \pi} \boldsymbol{v}\right)
$$

and has been used the vectorial identity [3] [11]

$$
(v \cdot \operatorname{grad}) v=\operatorname{grad}\left(\frac{1}{2} v^{2}\right)-v \times \operatorname{rot} v
$$

and the fact that [11]

$$
v \cdot(v \times \text { rotv }) \equiv(v \times v) \cdot \operatorname{rot} v=0 .
$$

By substituting (51) in (47) it's easy to see that

$$
\begin{aligned}
\frac{\partial}{\partial t}\left(\frac{1}{2} \rho v^{2}+\rho \varepsilon+\frac{H^{2}}{8 \pi}\right)= & -\operatorname{div}\left[\rho \boldsymbol{v}\left(\frac{1}{2} v^{2}+\omega^{\prime}\right)\right]+\boldsymbol{v} \cdot \boldsymbol{F}_{v}+\rho T \frac{\mathrm{d} s}{\mathrm{~d} t} \\
& +\frac{1}{4 \pi} \boldsymbol{H} \cdot[\nabla \times(\boldsymbol{v} \times \boldsymbol{H})]+\frac{c^{2}}{16 \pi^{2} \sigma} \boldsymbol{H} \cdot\left(\nabla^{2} \boldsymbol{H}\right) .
\end{aligned}
$$

From Equation (50) the following is obtained

$$
\boldsymbol{v} \cdot \boldsymbol{F}_{v}=\operatorname{div}\left(\boldsymbol{v} \cdot \tilde{\sigma}^{\prime}\right)-\sigma_{i j}^{\prime} \frac{\partial v^{i}}{\partial x^{j}}
$$

where the previous approximation with relation to the mass density has been used and an integration by parts has been done. Substituting in relation (46) all the results previously obtained one gets

$$
\begin{aligned}
& \rho T \frac{\mathrm{d} s}{\mathrm{~d} t}-\operatorname{div}(\kappa \boldsymbol{g r a d} T)-\sigma_{i j}^{\prime} \frac{\partial v^{i}}{\partial x^{j}}+\frac{1}{4 \pi} \boldsymbol{H} \cdot[\nabla \times(\boldsymbol{v} \times \boldsymbol{H})]+\frac{c^{2}}{16 \pi^{2} \sigma} \boldsymbol{H} \cdot\left(\nabla^{2} \boldsymbol{H}\right) \\
& =-\frac{1}{4 \pi} \nabla \cdot[\boldsymbol{H} \times(\boldsymbol{v} \times \boldsymbol{H})]+\frac{c^{2}}{16 \pi^{2} \sigma} \nabla \cdot[\boldsymbol{H} \times(\nabla \times \boldsymbol{H})] .
\end{aligned}
$$


Now, let $\boldsymbol{b}=\boldsymbol{v} \times \boldsymbol{H}$ be any vector, so that

$$
-\frac{1}{4 \pi} \nabla \cdot[\boldsymbol{H} \times(\boldsymbol{v} \times \boldsymbol{H})]=\frac{1}{4 \pi} \boldsymbol{H} \cdot[\nabla \times(\boldsymbol{v} \times \boldsymbol{H})]
$$

where the following vectorial identity [11] has been used

$$
\nabla \cdot(\boldsymbol{a} \times \boldsymbol{b})=\boldsymbol{b} \cdot(\nabla \times \boldsymbol{a})-\boldsymbol{a} \cdot(\nabla \times \boldsymbol{b})
$$

plus the fact that

$$
(\boldsymbol{v} \times \boldsymbol{H}) \cdot(\nabla \times \boldsymbol{H})=0
$$

because they are orthogonal vectors, and its scalar product is zero.

If in the second term of the right hand side member of (55) the previous vectorial identity is used again along with the following expression

$$
\nabla \times(\nabla \times \boldsymbol{H})=\nabla \times(\nabla \cdot \boldsymbol{H})-\nabla^{2} \boldsymbol{H}=-\nabla^{2} \boldsymbol{H}
$$

we simply get,

$$
\nabla \cdot[\boldsymbol{H} \times(\nabla \times \boldsymbol{H})]=(\boldsymbol{r o t} \boldsymbol{H})^{2}+\boldsymbol{H} \cdot\left(\nabla^{2} \boldsymbol{H}\right)
$$

just because $\nabla \cdot \boldsymbol{H}=0$. In this case, in (55) we get

$$
\rho T\left[\frac{\partial s}{\partial t}+\boldsymbol{v} \cdot \operatorname{grad} s\right]=\sigma_{i j}^{\prime} \frac{\partial v^{i}}{\partial x^{j}}+\operatorname{div}(\kappa \operatorname{grad} T)+\frac{c^{2}}{16 \pi^{2} \sigma}(\boldsymbol{r o t} \boldsymbol{H})^{2} .
$$

This is the general equation of heat transfer for MHD [5] [7]. On the other hand, according to the approximations already done, it is clear that in (59) the following equation is obtained

$$
\frac{J^{2}}{\sigma}=\frac{c^{2}}{16 \pi^{2} \sigma}(\text { roth })^{2} ;
$$

where $j^{2} / \sigma$ is the Joule heat per unit volume [5] [7]. However, since $\operatorname{rot} \boldsymbol{h}=0$ as we have seen before, then the value of the previous relationship is zero.

Then, in (59) we have that

$$
\rho T\left[\frac{\partial s}{\partial t}+\boldsymbol{v}^{\prime} \cdot \operatorname{grad} s\right]=S_{i j}^{\prime} \frac{\partial v^{\prime i}}{\partial x^{j}}+\operatorname{div}\left(\kappa \operatorname{grad} T^{\prime}\right)
$$

In fact, because we can write that $\boldsymbol{v}=\boldsymbol{v}_{o}+\boldsymbol{v}^{\prime}$ with $\boldsymbol{v}_{o} \gg \boldsymbol{v}^{\prime}$. But we saw that $\boldsymbol{v}_{o}=0$, so $\boldsymbol{v}=\boldsymbol{v}^{\prime}$. Besides

$$
S_{i j}^{\prime}=\eta\left[\frac{\partial v^{\prime i}}{\partial x^{j}}+\frac{\partial v^{\prime j}}{\partial x^{i}}-\frac{2}{3} \delta_{i j} \frac{\partial v^{\prime \ell}}{\partial x^{\ell}}\right]+\zeta \delta_{i j} \frac{\partial v^{\prime \ell}}{\partial x^{\ell}}
$$

is the corresponding viscosity stress tensor in terms of the perturbation in the velocity field. Equation (61) is the general equation of heat transfer for any fluid in terms of the perturbed quantities.

\section{The Magnetic Field of the Sunspots}

According to the previously given arguments, the velocity of the fluid inside the spots is damped, due to the stiffness in the fluid conferred by the magnetic viscosity which in turn is created by the magnetic field responsible for the generation of the spots; so that it is possible to assume that inside those regions the magnitude of the fluid velocity is smaller than the speed of sound in that continuous medium. Therefore, it could be thought that variations in hydrostatic pressure occurring as a consequence of the motion are so small, that any changes in density and in other thermodynamic quantities involved should be neglected. Nevertheless, it is clear that the solar fluid is subject to nonuniform heating so that its density undergoes changes which cannot be ignored, so that it is not possible to say that it is a constant. In consequence, it can be shown that [2]

$$
\rho T\left[\frac{\partial s}{\partial t}+\boldsymbol{v}^{\prime} \cdot \operatorname{grad} s\right]=\rho c_{p}\left[\frac{\partial T^{\prime}}{\partial t}+\boldsymbol{v}^{\prime} \cdot \operatorname{grad} T^{\prime}\right]
$$


where

$$
c_{p}=T\left(\frac{\partial s}{\partial T}\right)_{p}
$$

is the specific heat of the fluid at constant pressure [12]. Then, from Equation (64) the following is obtained

$$
\left[\frac{\partial T^{\prime}}{\partial t}+\boldsymbol{v}^{\prime} \cdot \operatorname{grad} T^{\prime}\right]=\chi \nabla^{2} T^{\prime}+\frac{1}{\rho c_{p}} S_{i j}^{\prime} \frac{\partial v^{\prime i}}{\partial x^{j}}
$$

with

$$
\chi \equiv \frac{\kappa}{\rho c_{p}}
$$

being the thermometric conductivity of the fluid [3].

As it is easy to see from (62), $S_{i j}^{\prime}$ is a second order term in the approximations. On the other hand, $\boldsymbol{v}^{\prime}$ is a small quantity and if the distances are relatively large, the gradient of the velocity field are very small. Then, the term $S_{i j}^{\prime} \partial v^{\prime i} / \partial x^{j}$ is even smaller. In that case, the viscous term in (65) can be neglected in comparison with the other quantities because it is a higher order term; with this the following result is obtained

$$
\frac{\mathrm{d} T^{\prime}}{\mathrm{d} t}-\chi \nabla^{2} T^{\prime}=0 \text {. }
$$

This is the final form of the general equation of heat transfer for the full process of generation, duration and disappearance of the solar Spots. On the other hand, since $T^{\prime}$ has been assumed to depend on the coordinates only, $\partial T^{\prime} / \partial t=0$; and in the previous relationship the following is obtained

$$
\frac{\partial}{\partial x^{i}}\left[T^{\prime} v^{\prime i}-\chi \operatorname{grad}_{i} T^{\prime}\right]=0
$$

where an integration by parts has been made and the term $T^{\prime} \partial v^{\prime i} / \partial x^{i}$ has been neglected because is a term of higher order in the approximations. The previous equation can be integrated in order to obtain

$$
\chi \operatorname{grad} T^{\prime}=T^{\prime} \boldsymbol{v}^{\prime},
$$

where the integration constant has been assumed to be zero without losing of generality. Now, and according to expression (41)

$$
\operatorname{grad} T^{\prime}\left[\chi-\gamma T^{\prime} t^{\prime}\right]=-\left[\alpha \boldsymbol{g} T^{\prime}+\boldsymbol{g r a d} p_{M}^{o}\right] T^{\prime} t^{\prime}
$$

Let's assume that for the condition of dynamic equilibrium, the thermometric conductivity can be neglected, so that only the other term is preserved. This is due to the fact that it could be very interesting to observe the phenomenon of the solar Spots as if it were frozen in time. Generally speaking, it is not possible to make such approximation as it will be seen in the next sections. Nevertheless, if for the moment that term is ignored, the following result is obtained in (70)

$$
\operatorname{grad} p_{M}^{o}=\gamma \operatorname{grad} T^{\prime}-\alpha \boldsymbol{g} T^{\prime}
$$

This is the analytical expression for the process of dynamic equilibrium in the solar spots. If only the z-component of the previous equation is considered, we get again that $\boldsymbol{g}=-\boldsymbol{k g}$; with $\boldsymbol{k}$ the unitary vector along the Z-axis of the inertial frame of reference used and of course, $g$ is the average constant value of the gravity at the surface of the Sun. In that case, it is proposed that the convective movements in the spots can be damped by magnetic regulation thus ensuring their permanency in the solar disk, if the following condition of dynamic equilibrium is satisfied

$$
\left|\operatorname{grad}_{z} h^{2}\right|>8 \pi \rho_{o}\left[\left|\gamma \operatorname{grad}_{z} T^{\prime}\right|+\alpha g T^{\prime}\right]
$$

Now, from the z-component of relation (71) the following is obtained

$$
\frac{\mathrm{d}}{\mathrm{d} z}\left[\gamma T^{\prime}-p_{M}^{o}\right]=-\alpha g T^{\prime}
$$


in which case

$$
h^{2}=8 \pi \rho_{o} T^{\prime}(\gamma+\alpha g z)
$$

where $z$ is any characteristic height of the photosphere in the region where the solar spots are produced. From the previous relationship, the following expressions can be obtained

$$
\boldsymbol{h}_{+}=\boldsymbol{k} \lambda_{+}
$$

and

$$
\boldsymbol{h}_{-}=-\boldsymbol{k} \lambda
$$

where

$$
\lambda_{+}=\left[8 \pi \rho_{o} T^{\prime}(\gamma+\alpha g z)\right]^{1 / 2}
$$

and

$$
\lambda_{-}=-\left[8 \pi \rho_{o} T^{\prime}(\gamma+\alpha g z)\right]^{1 / 2}
$$

In (75) and (76), $\boldsymbol{h}_{+}$and $\boldsymbol{h}_{-}$are two magnetic fields which have opposite polarity. Thus, the permanence of the solar Spots on the surface of the Sun is assured if the magnitude of the magnetic field for each member in the couple is proposed to have the following value

$$
h_{+}=2\left[2 \pi \rho_{o} T^{\prime}(\gamma+\alpha g z)\right]^{1 / 2}
$$

and

$$
h_{-}=2\left[2 \pi \rho_{o} T^{\prime}(\gamma+\alpha g z)\right]^{1 / 2}
$$

Clearly, both magnetic fields have the same magnitude. On the other hand, from (76) and (78) it is easy to see that $\boldsymbol{h}_{-}=\boldsymbol{k} \lambda_{+}$; so that those fields are also oriented in the same direction. In consequence, if $\boldsymbol{h}_{+}$is a magnetic field with (N-S) polarity; $\boldsymbol{h}_{-}$will have the opposite polarity; that is (S-N). It would look as if these were the poles of a horseshoe shaped magnet.

It can be seen from Equation (71) that as the intensity of the magnetic field generating the solar spots weakens its influence declines in favour of the thermal gradient basically because the other term is a constant for a particular couple of spots. Under such circumstances, the conditions for the startering mechanism of thermal convection are given; so that thermal processes become more important taking over the situation little by little. Again, in the limit when $\boldsymbol{h}=0$, we have that

$$
\frac{\mathrm{d} T^{\prime}}{\mathrm{d} z}=-\frac{\alpha g T^{\prime}}{\gamma}
$$

As usual [2] [3], the condition to generate the convective movements that mark the disappearance of the solar spots turns out to be similar to that proposed in the introductory paragraph, that is to say that, the magnitude of the thermal gradient be greater than a given value; this is

$$
\left|\frac{\mathrm{d} T^{\prime}}{\mathrm{d} z}\right|>\frac{\alpha g T^{\prime}}{\gamma}
$$

Finally, integrating Equation (81) the following result is obtained

$$
T^{\prime}=T_{o} \exp \left[-\frac{\alpha g z}{\gamma}\right]
$$

This relation gives the temperature at which the thermal convection starts. Here, $T_{o}$ is the characteristic temperature of the solar spots. According to some numerical data from specialized literature, it is possible to make an approximate calculation of that temperature. For example let $T_{o}=3700 \mathrm{~K} ; \alpha=0.5 \times 10^{-4} \mathrm{~K}^{-1} ; g=2.74 \times 10^{4}$ $\mathrm{cm} / \mathrm{seg}^{2} ; z=8 \times 10^{7} \mathrm{~cm} ; \gamma=2.4 \times 10^{8} \mathrm{~cm}^{2} / \mathrm{K} \cdot \mathrm{seg}^{2}$. In this case, from (83) we obtain $T^{\prime}=2344 \mathrm{~K}$; so that $T=T_{o}+$ $T^{\prime}=6.044 \mathrm{~K}$ which is the approximate average temperature of the solar photosphere. Additionally, and with the help of the previous data, it is easy to see that convective movements are generated when 


$$
\left|\frac{\mathrm{d} T^{\prime}}{\mathrm{d} z}\right|>1.32 \times 10^{-5} \mathrm{~K} / \mathrm{cm}
$$

Finally, considering that if $\rho=10^{-5} \mathrm{gr} / \mathrm{cm}^{3}$ [4], the value of density inside the Spots would have to be slightly smaller than that in the photosphere, according to the decomposition proposed in paragraph 2 of this paper. In this case assuming that for the interior of the spots, $\rho_{o}=0.715 \times 10^{-6} \mathrm{gr} / \mathrm{cm}^{3}$, then,

$$
h=3837 \text { gauss; }
$$

as it is easy to see from (79) or (80). Certainly, the obtained value is within the order of magnitude of the magnetic fields of the solar spots measured with the aid of the Zeeman Effect [4] [10] [13].

\section{Persistency of the Sunspots}

From the Z-component of relation (70) the following result can be obtained

$$
t^{\prime}=\frac{\chi q}{T^{\prime}\left[\gamma q-\frac{\mathrm{d} p_{M}^{o}}{\mathrm{~d} z}+\alpha g T^{\prime}\right]}
$$

where

$$
q \equiv \frac{\mathrm{d} T^{\prime}}{\mathrm{d} z}
$$

is the thermal gradient in the Z-direction.

Persistence of the Sunspots seems to depend mainly on the z-components of both the thermal gradient and the gradient of the square of the magnetic field which generates them. Nevertheless, the dominant mechanism at the beginning of the phenomenon is that of magnetic regulation of the convective movements; while at the end of it, disappearance of the Spots is determined by the predominance of the thermal gradient over the other quantities. In consequence it seems reasonable to assume that the average life time of the Sunspots can be determined from the dynamic equilibrium condition between the magnetic regulation and starting mechanisms of thermal convection; so that for the case in which $\boldsymbol{F}_{\mathrm{MHD}}=0$, in (86) we only have

$$
\tau \approx \frac{\chi q}{\alpha g T^{\prime 2}}
$$

which is not other than the average life time of the spots. The previous relationship must at least provide an order of magnitude for the time duration of the Sunspots. Astronomical observations of the phenomenon made by other researchers show that the average life time of the phenomenon could be of days, weeks or even several months [4]. In general, it is asserted that the average life time is somewhat greater than one solar revolution [1]; this is, approximately 28 days. Let's consider for example that for the most superficial layers of the sun's photosphere, $\chi=10^{18} \mathrm{~cm}^{2} / \mathrm{seg}$. Then, with the numerical data from the previous paragraph and with $q=2 \times 10^{-5}$ $\mathrm{K} / \mathrm{cm}[4]$ the following is obtained

$$
\tau \approx 31 \text { days }
$$

Obviously, the permanency in time of a particular couple of spots in the solar disk, depends on the difference between its temperature $T_{o}$ and that of the photosphere, $T$; this is, it depends on $T^{\prime}$. Thus, the higher the spots temperature, the smaller the $T^{\prime}$, and consequently, the larger the spot average life time.

\section{Conclusions and Discussion}

From the results obtained, it can be assumed that the Sunspots are phenomena very related with processes occuring on the surface of the sun, at a very shallow depth in the solar photosphere; that is, they are basically surface phenomena. Generation, evolution and disappearance of the Sunspots can be explained in terms of the competition established between two dynamic mechanisms: one of magnetic nature which controls and delays thermal convection, and the other one of thermal nature which propitiates convective movements. What happens there may be possibly as follows: the magnetic field characteristic of the solar spots starts to be created at any 
moment in certain regions of the photosphere. While its intensity increases, a growing magnetic viscosity is generated in the fluid which makes it stiff. Such stiffness damps thermal agitation in the fluid makes it difficult for the fluid to move in those regions. As a consequence, the temperature in those places decreases creating the required conditions for the appearance of a thermal gradient whose value is adjusted to the increasing value of the magnetic field intensity, being created. Thus, as the magnetic field intensity grows, the stiffness in the fluid increases and the magnitude of the thermal gradient grows bigger, firmly opposing the establishment of the said magnetic field. The fight between these two mechanisms leads to a gradual loss of thermal energy in those regions, resulting in areas which are darker and colder than their surroundings, which constitutes the Sunspots. On the other hand, the solar activity in those regions begins to grow and reaches its maximum value when the creation of the thermal and magnetic field gradients is completed.

Next an evolution period of the spots takes place, which is characterized by the existence of a state of dynamic equilibrium between both mechanisms, and by the fact that sun's activity in those regions is at its maximum. In that intermediate stage, the dominant force is the huge solar gravity which acts over the fluid. Throughout of this period, the hot fluid flows more or less parallel to the surface of the sun due to its gravitational attraction, diverging from the center of the spot and overflowing through its boundaries [10]. At the same time, different varieties of the so called faculae are produced depending on their size; that is, as sparks, torches and flames. It is possible that the origin of such things can be found in the splashing caused by thermal fluctuations in the fluid, which occurs at the edges of the spots.

In the final stage, the magnitude of the magnetic field begins to decrease; meanwhile the importance of the thermal processes increases until they become the dominant part when $\boldsymbol{h}=0$. At this moment, the starting mechanism of thermal convection is completed and violent convective movements begin, which mix the fluid inside the spots already free of its magnetic stiffness, with the fluid from the photosphere; thus equalizing the temperature in those regions with that of its surroundings. With that, the spots disappear at those latitudes and the solar activity in them is depleted.

Another interesting result explains the polarity of the Sunspots, as well as its tendency to appear as magnetic bipolar couples. Apparently, they can be considered as huge electromagnets produced by gigantic solenoids formed under the surface of the photosphere by the ionized fluid which rotates at high speed and creates the monstrous electric currents which generate and maintain the characteristic magnetic field in those regions. The following hypothesis is suggested by the polarity in the spots, their appearance, permanency in the solar disk and subsequent disappearance. The highly ionized solar fluid [13] must be basically formed by an inhomogeneous mixture of positive and negative ions; as well as by electrically neutral atoms. It can be assumed that throughout one solar cycle, the contents of positive ions are larger than that of negative ions; so that the electric current produced by such an excess of charge would determine the polarity of the spots in each hemisphere. On the other hand, and due to the fact that the surface where the spots appear is relatively small as those regions move through the solar disk, and at the end of a certain time which must coincide with their average life time, it can be expected that locally, the number of positive and negative ions which rotate in the solenoid become equal. Clearly, both kinds of ions must move in the same sense. As a positive particle in motion is equivalent to a negative particle which moves in the opposite direction [14], the net current in the solenoid is zero and, of course, the magnetic field is cancelled. This fact is enough to give a heuristic explanation about the origin, the duration and the disappearance of the spots, as well as their polarity and the end of the solar activity in those regions.

To explain the change in polarity at both hemispheres in each solar cycle, it's enough to assume that in sun's latitudes where the spots are usually observed, gigantic turbulent fluid flows take place, in a way similar to what occurs in the Earth at the cyclonic zones. The flows and the turbulent eddy in the northern hemisphere move in the opposite sense as they do in the southern hemisphere due to the Coriolis force [15]. If throughout one cycle, the highly ionized fluid has a net charge of a given sign, the polarity of the electromagnets will be inverted in one hemisphere with respect to the other one. Thus, for example, couples of spots in the northern hemisphere would have (N-S) polarity; while in the southern hemisphere their polarity would be (S-N). At the end of 11.5 years, a new cycle begins and that disparity is inverted, so that northern couples will have (S-N) polarity and the southern couples (N-S) polarity. This fact suggests that in one cycle the huge electric currents which feed the gigantic solenoids which create the magnetic field of the spots, are generated by an excess of charge of the same sign in both solar hemispheres; and in the following cycle, by an excess of charge of the opposite sign. The question is: what would be the physical mechanism which originated such change in polarity with so much re- 
gularity and in such a short time? The following argument is considered. Due to the high temperatures and pressures in the deep regions of the sun, the conditions are given for the production of the solar plasma, basically formed by a mixture of positive and negative ions. Assume that the highly ionized fluid under the influence of a tremendous thermal stirring is slowly pushed against the solar gravity and the disorderly thermal movement, towards regions of the photosphere until the turbulent tropical regions of the sun, is reached. There, they would feed the solenoids responsible for the production of the intense magnetic fields of the couples of Sunspots.

The transport of ions from the convective zone up to the surface of the sun, is responsible of removing both the huge quantity of heat produced and products of the combustion process, while at the same time, feeding with new fuel the thermonuclear oven [10], it can be the responsibility of the powerful shock waves coming from that zone and from the oven itself. However, shock waves would drag positive and negative ions in a different way; that difference depending on the mass of each type. In fact, even in the case of ionized hydrogen $\left(\mathrm{H}^{+}\right)$, the difference between its mass and that of the free electron $\left(\mathrm{e}^{-}\right)$produced during the process of ionization is around 2000. For other elements ionized by the loss of one or more orbital electrons, that difference could be even larger; such was the case of $\left(\mathrm{He}^{+}\right)$whose mass could be four times bigger than that of $\left(\mathrm{H}^{+}\right)$. Then, it could be expected the migration of positive ions to be slower than that of negative ions; and such difference could make the former to reach the photosphere with a delay of 11.5 years with respect to the latter, in each Solar Cycle.

Thus, in one cycle there could be surges of ionized fluid mainly of negative charge and in the following, mainly of positive charge. This last argument is only one hypothesis derived from the results previously obtained, and is subjected to validation. It is put forward here as an heuristic attempt to explain the above mentioned phenomenon, because it is difficult to imagine some other processes occurring inside the sun that can have so regular effects. Nevertheless, maybe one could have an indirect proof of the periodicity and origin of this phenomenon by determining whether in the solar wind of the present solar cycle there is excess of a certain type of charge reaching the Earth and coming from both hemispheres of the sun, and if the sign of that charge coincides with the present polarity of the spots. The next step would be to measure whether in the next cycle also there is the said excess of charge and if it is of the opposite sign than the excess of charge of the previous cycle. If the answer is affirmative, the polarity of the spots in both hemispheres has to be as expected and, clearly, it must be opposite to that of the previous cycle. If the previous hypothesis is proven, the problem of finding a theoretical solution to the phenomenon of ionic migration in a star like the sun remains unsolved. Such task will be attempted in the near future. In the opposite case, another possible explanation would be searched for.

\section{References}

[1] Wilson, P.R. (1994) Solar and Stellar Activity Cycles. Cambridge Astrophysics Series: 24. Cambridge University Press, Cambridge. http://dx.doi.org/10.1017/cbo9780511564833

[2] Fierros Palacios, A. (1994) La generación de un estímulo atmosférico y el problema de la contaminación del aire en el Valle de México. Rev. del IMP, XXVI, 47-55.

[3] Landau, L.D. and Lifshitz, E.M. (1959) Fluid Mechanics. Addison-Wesley Publishing Co.

[4] Cowling, T.G. (1968) Magnetohidrodinámica. Editorial Alhambra, S.A., Madrid, Buenos Aires, México.

[5] Landau, L.D. and Lifshitz, E.M. (1960) Electrodynamics of Continuous Media. Addison-Wesley Publishing Co.

[6] Fierros-Palacios, A. (1992) Las ecuaciones de un fluido viscoso a partir de un principio variacional tipo Hamilton. Revista Mexicana De Fisica, 38, 518-531.

[7] Fierros Palacios, A. (1998) Las ecuaciones diferenciales de campo de la magnetohidrodinámica. Revista Mexicana De Fisica, 44, 120-127.

[8] Fierros-Palacios, A. (1993) La ecuación de campo para la densidad de masa. Rev. del IMP, XXV.

[9] Landau, L.D. and Lifshitz, E.M. (1959) Theory of Elasticity. Addison-Wesley Publishing Co.

[10] Gamow, G. (1967) Una estrella llamada Sol. Espasa Calpe, S.A., Madrid.

[11] Spigel, M.R. (1959) Vector Analysis and an Introduction to Tensor Analysis. Schaum Publishing Co., New York.

[12] Landau, L.D. and Lifshitz, E.M. (1958) Statistical Physics. Addison-Wesley Publishing Co.

[13] Unsöld, A. (1979) El Nuevo Cosmos. Siglo Veintiuno Editores, S.A.

[14] Resnick, R. and Halliday, D. (1966) Physics. John Wiley and Son, Inc., New York, London, Sydney.

[15] Goldstein, H. (1959) Classical Mechanics. Addison-Wesley Publishing Co., Inc. 
[16] Fierros Palacios, A. (1997 y 1998) El principio tipo Hamilton en la Dinámica de los Fluidos. Primera y segunda ediciones. McGraw-Hill, Caps, VIII y XI.

[17] Fierros Palacios, A. (2006) The Hamilton-Type Principle in Fluid Dynamics. Fundamentals and Applications to Magnetohydrodynamics, Thermodynamics, and Astrophysics. Springer-Verlag, Wien.

[18] Reif, F. (1965) Fundamentals of Statistical and Thermal Physics. International Student Edition, McGraw-Hill, London, New York.

\section{Appendix}

The influence of the external magnetic field on the system must be taken into account in order to determine its thermodynamic state [16]. In particular, the thermodynamic pressure can be written as follows [7] [16]

$$
p(\rho, T, \boldsymbol{H})=c_{o}^{2} \rho+\frac{\beta}{k} T+\frac{H^{2}}{8 \pi}
$$

where

$$
c_{o}^{2}=\left(\frac{\partial p}{\partial \rho}\right)
$$

is the square of the velocity of sound in the medium, $\rho(x, t)$ is the mass density and $T$ the temperature. Besides $H^{2} / 8 \pi$ is the hydrostatic magnetic pressure [7] [16]; since

$$
\frac{\beta}{k}=\left(\frac{\partial p}{\partial T}\right)
$$

with

$$
\beta=\frac{1}{V}\left(\frac{\partial V}{\partial T}\right)
$$

the coefficient of volumetric expansion and

$$
k=-\frac{1}{V}\left(\frac{\partial V}{\partial p}\right)
$$

the isothermic compressibility which is always positive [17] [18].

On the other hand, it is affirmed in astrophysics that the solar plasma behaves as an ideal gas from the thermodynamic point of view; so that the equation $p=R \rho T$ must be fulfilled; with $R$ being the universal gas constant. In that case, in (A-1) we have that

$$
p_{i}(\rho, T)=R^{\prime} \rho T
$$

where $\rho(x, t)$ is the mass density and $T(x, t)$ the temperature, in such a way that

$$
p_{i}(x, t) \equiv p-\frac{H^{2}}{8 \pi}
$$

and $R^{\prime}=2 R$. Let's consider that the hydrostatic pressure in the interior region of the solar spots is such that it satisfies the hydrostatic Equation (17); this is,

$$
p_{i}(\boldsymbol{x}, t)=\rho_{o} \boldsymbol{g} \cdot \boldsymbol{x}+\text { constant } ;
$$

where $\boldsymbol{g}$ is the acceleration of the gravity on the surface of the Sun. If the gradient is calculated from expression (A-7), the relationship (A-8) is used and the fact that $\rho=\rho_{o}+\rho^{\prime}$ with $\rho_{o}$ a constant and $\rho^{\prime} \ll \rho_{o}$ a small variation in the mass density is taken into account, $\rho_{o} \boldsymbol{g}=\rho \boldsymbol{g}-\rho^{\prime} \boldsymbol{g} \approx \rho \boldsymbol{g}$; because it is the dominant term, Equation (1) is obtained. 